\title{
Enhanced multi-exciton emission property in gradient alloy core/shell CdZnSeS/ZnS quantum dots: balance between surface passivation and strain-induced lattice defect
}

Hongyu Yang ${ }^{\dagger}$, Lei Zhang ${ }^{\dagger}$, Ying Tang ${ }^{\dagger}$,Wenbin Xiang ${ }^{\dagger}$, Xiaoyong Wang ${ }^{*,+}$, Min Xiao ${ }^{\dagger}, \quad s$ , Yiping Cuił, Jiayu Zhang*,

${ }^{\dagger}$ Advanced Photonics Center, Southeast University, Nanjing 210096, Jiangsu, China National Laboratory of Solid State Microstructures, College of Engineering and Applied Sciences, and School of Physics, Nanjing University, Nanjing 210093, China

$\S$ University of Arkansas, Fayetteville, Arkansas 72701, United State

*Corresponding author:

Email: wxiaoyong@nju.edu.cn

Email: jyzhang@seu.edu.cn

\section{$\underline{\text { Table of content }}$}

Figure S1. Single-dot fluorescence traces of \#core, \#6ML and \#12ML. S2

Figure S2. $\mu_{o n}$ and $\mu_{\text {off }}$ as function of shell thickness. $\quad$ S3

Figure S3. PL decay traces of \#core\#,6ML, \#9ML and \#12ML as function of average exciton. S4

Figure S4. ASE spectra of \#core\#,6ML, \#9ML and \#12ML. S5 

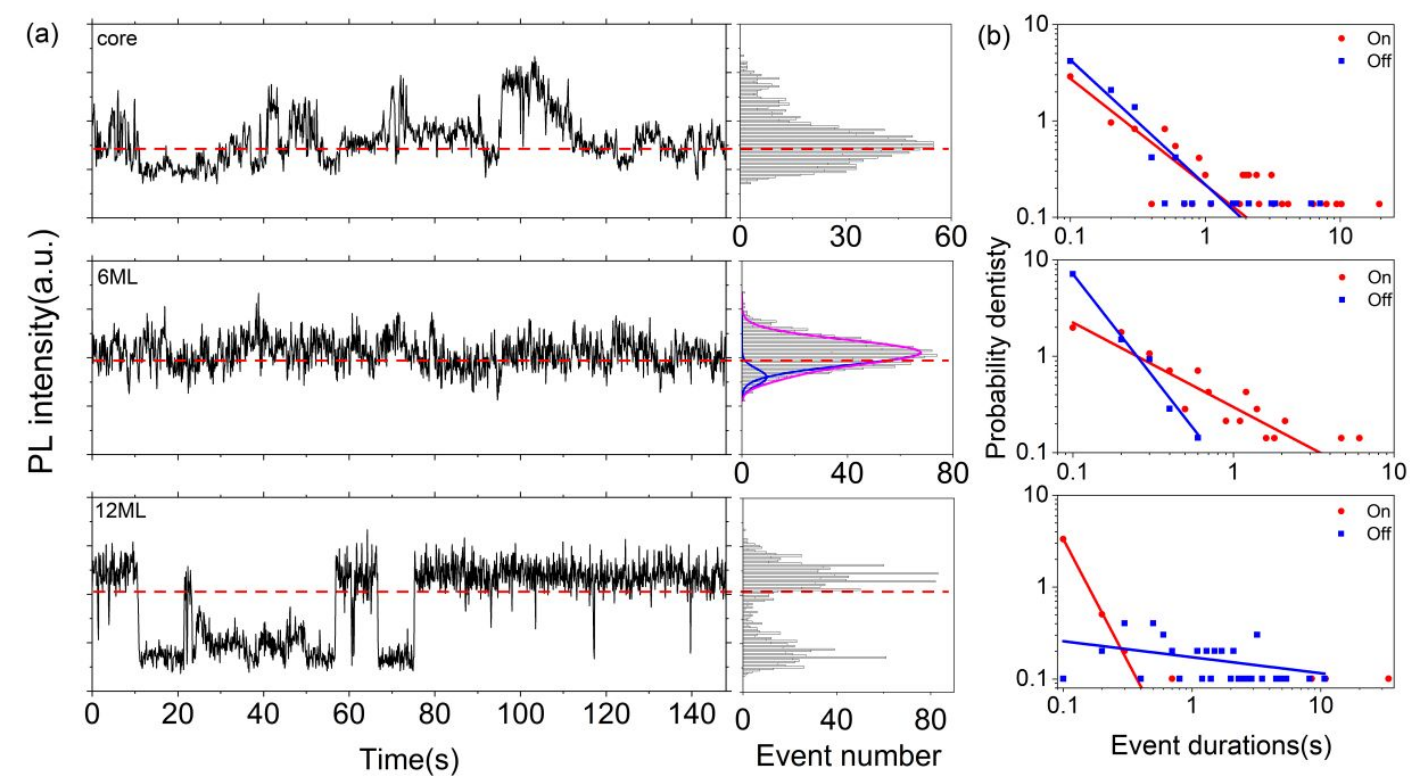

Figure S1 (a) Typical single-dot fluorescence traces of \#core, \#6ML and \#12ML sample under excitation of $405 \mathrm{~nm}$ pico-second pulses, the right show intensity distribution histograms. The threshold set $15 \%$ lower than the peak of "on" states were depicted using the red dashed-line. (b) corresponding Log-Log plot of $P_{\text {on }}$ and $P_{\text {off }}$ distributions. 


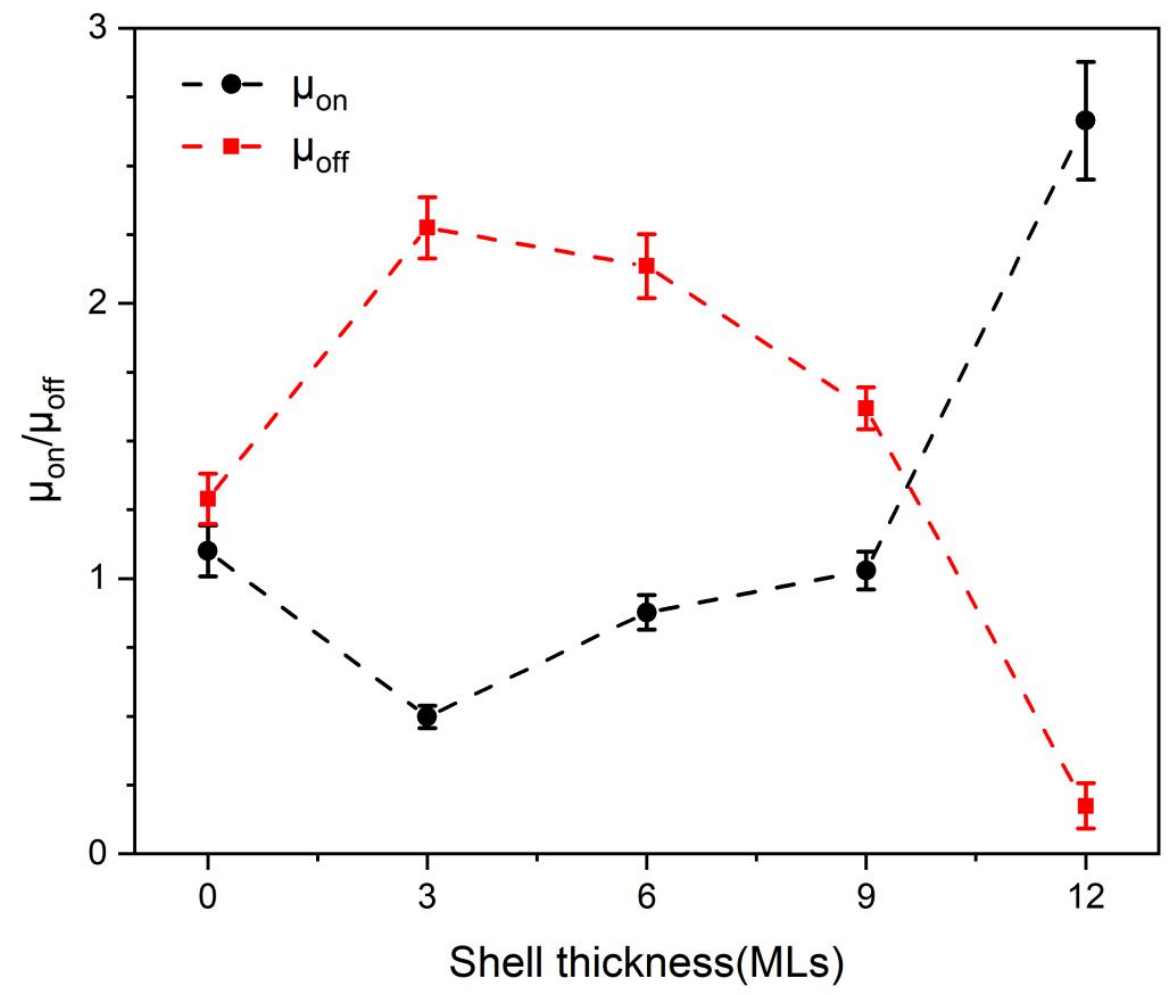

Figure S2. Event duration probability fitted results of $\mu_{\text {on }}$ and $\mu_{\text {off }}$ as function of shell thickness. 

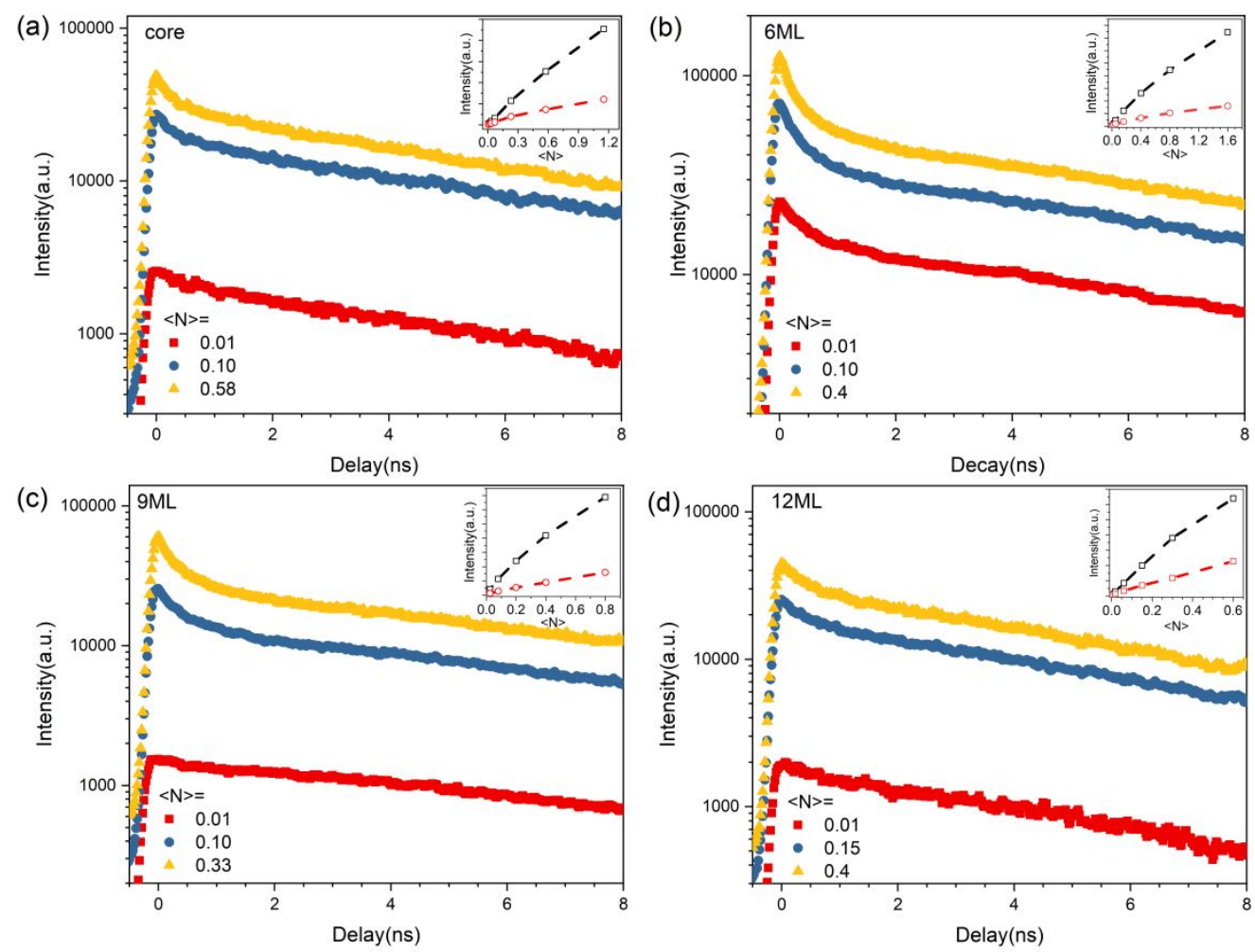

Figure S3 (a)\#core (b) \#6ML (c) \#9ML (d) \#12ML PL decay traces as function of average exciton, the inset shows the PL intensity at delay time $t=0$ and $5 \mathrm{~ns}$, respectively. 

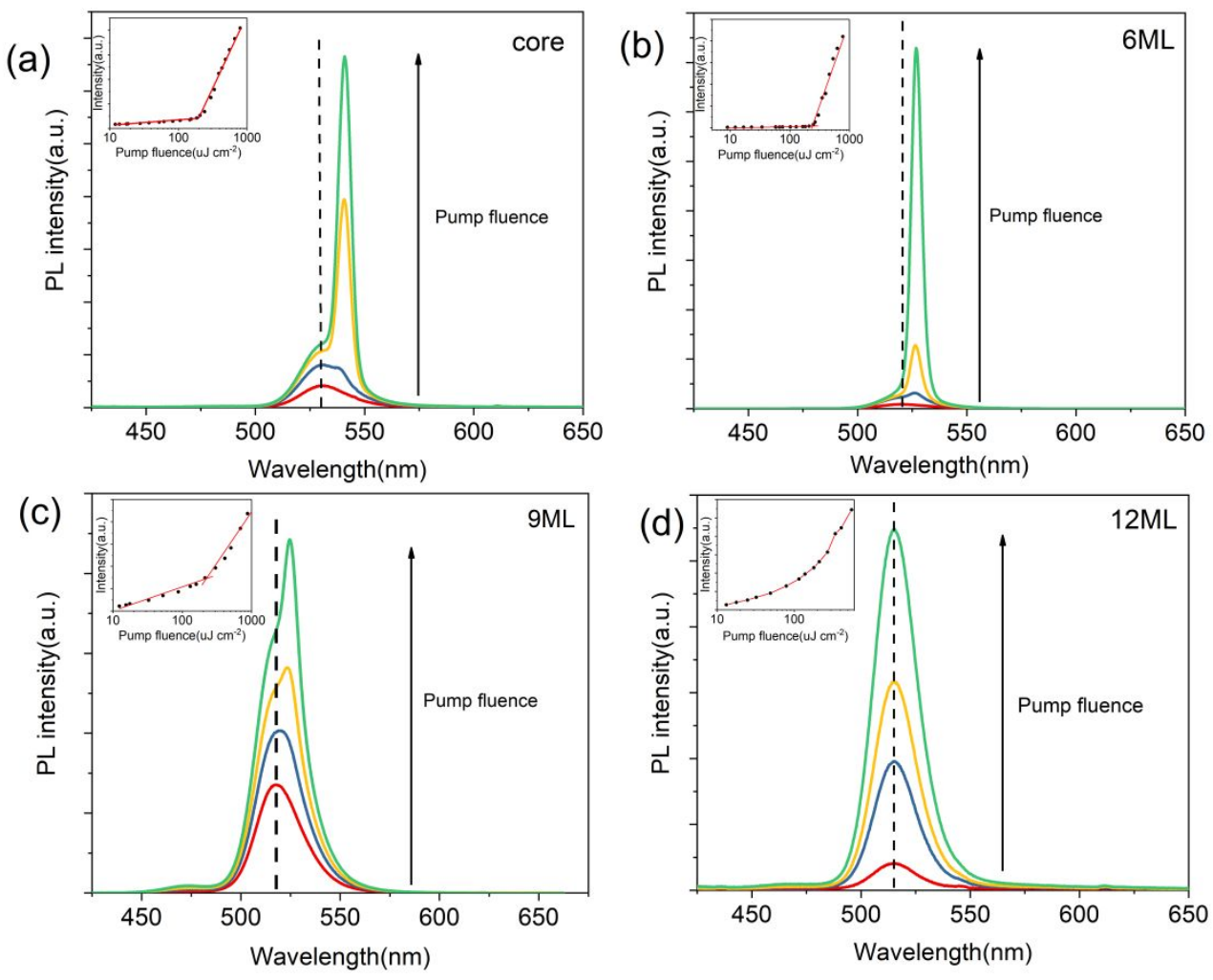

Figure S4 Pump-fluence-dependent ASE spectra of (a) \#core (b) \#6ML (c) \#9ML (d) \#12ML QD film. The inset presents the evolution of the integrated emission intensity as a function of the pump fluence. The black dash line shows the peak of spontaneous emission. 